\title{
Quantification of Metal-Rich Minerals in Ore Using X-Ray Micro-CT.
}

\author{
Frederik Coppens ${ }^{1}$ and Bart Pauwels ${ }^{2}$. \\ 1. Applications Department, Bruker microCT, Kontich, Belgium. \\ 2. Research and Development Department, Bruker microCT, Kontich, Belgium.
}

The metallic minerals inside a sample of ore from a commercial mine are analysed by means of X-ray micro-CT. It concerns a sample from a copper-gold porphory deposit, the main copper mineral is associated with pyrite. The aim is to obtain information about the amount, size and distribution of the metallic inclusions.

Determining volumetric mineralogical characteristics is one of the key issues in commercial geology. The advantage micro-CT poses over other chemical characterization techniques is that it delivers 3D information [1] in a non-destructive manner without the need for otherwise lengthy sample preparation. Micro-CT, in combination with other quantitative methods (i.a. XRD, XRF, AAS, ICP-MS,...), allows to determine the amount of contained metal, cut-off grades and other parameters related to a mining feasibility study or operation. Moreover the combination of micro-CT with micro-XRF holds the promise of providing 3D elemental information [2] and [3].

The sample was scanned using a SkyScan 1173 machine (Bruker microCT, Kontich, Belgium). Of the $\sim 60 \mathrm{mmx} 30 \mathrm{~mm} 25 \mathrm{~mm}$ sample the top half was scanned using a voltage of $130 \mathrm{kVp}(61 \mathrm{uA})$ and a $0.25 \mathrm{~mm}$ brass filter. The spatial resolution of the data is $15.71 \mathrm{um} /$ pixel for $2240 \times 2240$ lines. Frame averaging was set at 4 resulting in a total scan duration of $2 \mathrm{~h} 50 \mathrm{~m}$. The obtained data was reconstructed using a modified Feldkamp algorithm for cone beam projections; the software package used is NRecon (Bruker microCT, Kontich, Belgium) with GPUReconServer as reconstruction engine.

The obtained data can be used directly for visualization purposes; Figure 1 (left) shows a representative reconstructed slice. The different grey-scales correspond to different levels of attenuation for which the dominant contribution relates to differences in chemistry. The brightest particles are the metal-rich minerals, their quantification being the point of interest. Clearly differences in attenuation within the matrix and the different metal-rich particles themselves are present. These also reflect differences in chemistry, they are however outside the scope of the current review. Alternatively 3D volume rendering software, such as CTvox (Bruker microCT, Kontich, Belgium) can be employed. As a manner of example a screen shot using maximum intensity projection viewing mode is shown in Figure 1 (right), the metal-rich mineral particles are clearly discernible.

Analysis of the data was performed using the CTAnalyser software package (Bruker microCT, Kontich, Belgium). In order to obtain quantitative information a segmentation procedure is performed to binarize the greyscale images. An adaptive thresholding algorithm using the mean of minimum and maximum vales within a radius of 2 pixels in 3D was used for the metal-rich minerals. For the matrix a global threshold was selected manually. The structure thickness distribution of the metal-rich mineral particles was evaluated, the results are plotted in Figure 2 (left). Structure thickness evaluation provides a modelindependent measure of 3D thickness and is determined by local sphere-fitting for individual voxels. It shows a peak value around $94 \mu \mathrm{m}$, the average structure thickness was $141 \mu \mathrm{m}$. Individual 3D analysis of the particles indicates that with increasing volume the complexity of their shape increases significantly. 
This is illustrated in Figure 2 (right) where the sphericity is shown relative to the volume equivalent sphere diameter. This acquired information can prove useful in the assessment of mineral liberation fractions.

\section{References:}

[1] D. Koroteev et al, Micro-CT User meeting Abstract Book (2011), p. 40.

[2] P. Bruyndonckx et al, Proceedings of SPIEE: Developments in X-ray Tomograpghy VII (2010), p. 7804.

[3] A. Mutina et al, Micro-CT User Meeting Abstract Book (2012), p. 31

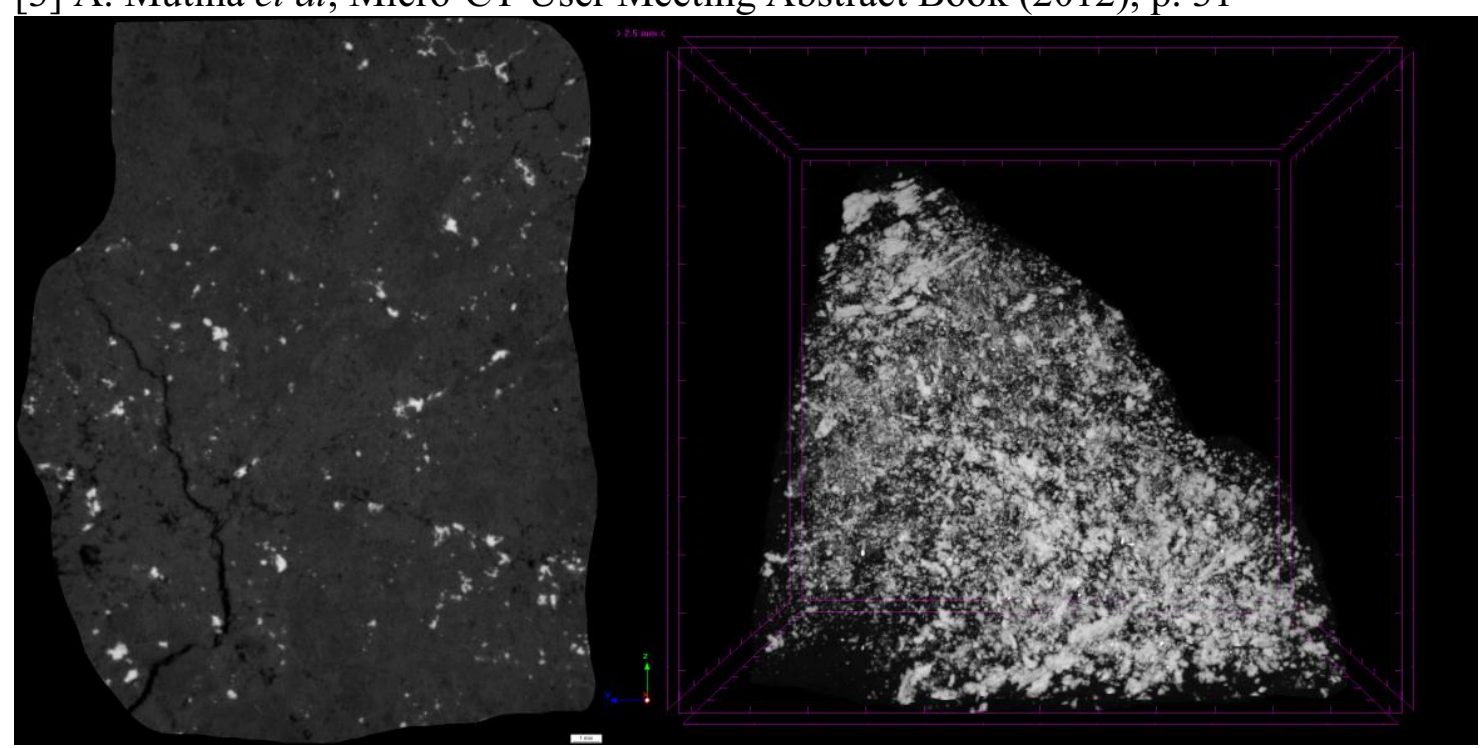

Figure 1. X-ray micro-CT data: XY slice of sample scanned at $15.7 \mu \mathrm{m}$ (left) and volume rendering image in Maximum Intensity Projection view mode (made using CTvox) (right).

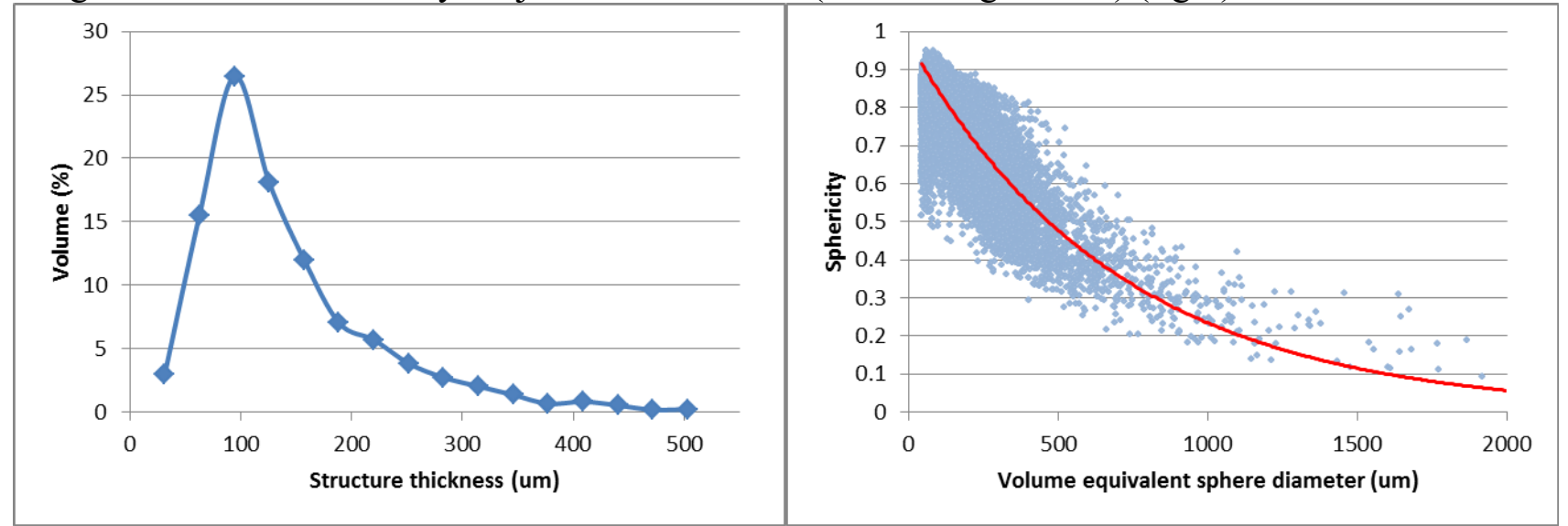

Figure 2. Structure thickness distribution of metal-rich particles (left) and sphericity vs. volume equivalent sphere diameter (right). 